\title{
Preparation and Synthetic Applications of Aryl Tetraflates $\left(\mathrm{ArOSO}_{2} \mathrm{CF}_{2} \mathrm{CF}_{2} \mathrm{H}\right)$
}

\author{
Vsevolod V. Rostovtsev, Lois M. Bryman, Christopher P. Junk, Mark A. Harmer, and Liane G. Carcani \\ DuPont Central Research and Development, Experimental Station, Wilmington, Delaware, 19880, USA \\ vsevolod.rostovtsev@usa.dupont.com
}

Table of Contents

General Considerations.

Synthetic Procedures for Palladium-Catalyzed Couplings and Characterization of

Products.

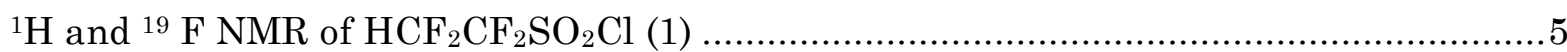

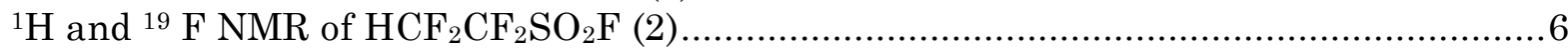

${ }^{1} \mathrm{H}$ and ${ }^{19} \mathrm{~F}$ NMR of $\left(\mathrm{HCF}_{2} \mathrm{CF}_{2} \mathrm{SO}_{2}\right)_{2} \mathrm{O}$ (3), small impurity is

$\mathrm{HCF}_{2} \mathrm{CF}_{2} \mathrm{SO}_{2} \mathrm{OCF}_{2} \mathrm{CF}_{2} \mathrm{H}$

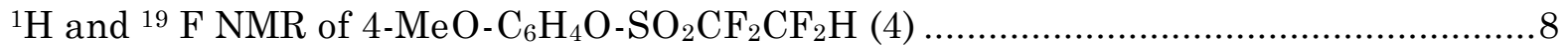

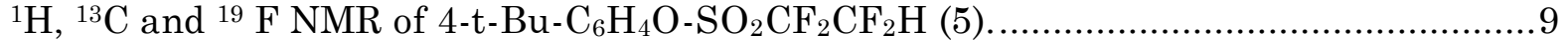

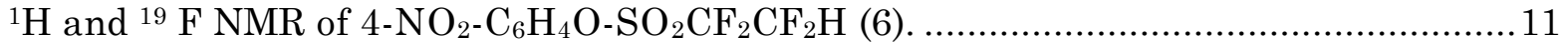

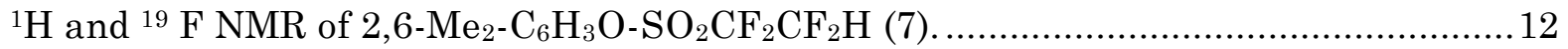




\section{General Considerations.}

${ }^{1} \mathrm{H}$ and ${ }^{19} \mathrm{~F}$ NMR spectra were recorded on a $400 \mathrm{MHz}\left({ }^{1} \mathrm{H}\right.$ frequency $\left.400.5524 \mathrm{MHz}\right)$ FT NMR instrument. The spectra were referenced to residual deuterated solvent peaks. $\mathrm{CFCl}_{3}$ was used as an internal standard for ${ }^{19} \mathrm{~F}$ NMR. IR spectra were recorded on an FT-IR spectrometer using pellet samples prepared using $\mathrm{KBr}$. Thermogravimetric analysis (TGA) is a technique that records the changes in mass of a sample in response to increase of temperature. It was carried out under nitrogen and temperature was increased at $1{ }^{\circ} \mathrm{C} / \mathrm{min}$ rate. Identity of known compounds was confirmed by comparison to published ${ }^{1} \mathrm{H}$ NMR data.

Catalyzed couplings were carried out under inert atmosphere (nitrogen). Anhydrous solvents were used as received, except for sulfolane, which was dried under vacuum at $120{ }^{\circ} \mathrm{C}$ for six hours. (catechol) $\mathrm{PCl}_{3}, \mathrm{Pd}_{2}(\mathrm{dba})_{3}, \mathrm{Pd}(\mathrm{OAc})_{2}, \mathrm{PBCy}_{2}, \mathrm{PCy}_{3}, \mathrm{Xanthos}, \mathrm{dppp}, \mathrm{P}_{4} \mathrm{O}_{10}, \mathrm{DBU}$, and pyridine were used as received. $\mathrm{Et}_{3} \mathrm{~N}$ was degassed and stored over $\mathrm{KOH}$ pellets. Aniline was passed through a short alumina plug and degassed before use. Inhibitor-free styrene was degassed and stored in the dark at $-20{ }^{\circ} \mathrm{C}$ under nitrogen. $o$-Tolylboronic acid from recrystallized from water prior to use. $\mathrm{CsF}$ and $\mathrm{KF}$ were dried at $120^{\circ} \mathrm{C}$ overnight under dynamic vacuum. Moisture sensitive materials were handled in a nitrogen-atmosphere glove box. All couplings were set up in the glove box. For Suzuki couplings, significantly lower yields of biphenyls were obtained when reactions were set up using standard bench-top techniques (following, for example, Procedure D from Littke et al., J. Am. Chem. Soc. 2000, 4020).

1,1,2,2-Tetrafluoroethanesulfonic acid (TFESA) was prepared as described in ref. 15 of the main text. It is now commercially available.

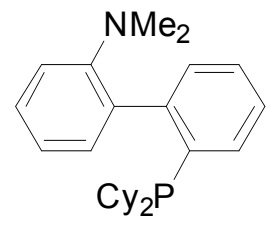

BPCy,<smiles>CC1(C)c2cccc(P)c2Oc2c(P(c3ccccc3)c3ccccc3)cccc21</smiles>

XantPhos

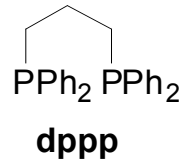

dppp 


\section{Synthetic Procedures for Palladium-Catalyzed Couplings and Characterization of Products.}

4-Methoxyphenyl-1,1,2,2-tetrafluoroethanesulfonate (4) was prepared following the procedure described for compound 5. Yield $3.25 \mathrm{~g}(94 \%)$. ${ }^{1} \mathrm{H} \mathrm{NMR}\left(400 \mathrm{MHz}, \mathrm{CDCl}_{3}\right): 3.79(3 \mathrm{H}, \mathrm{s}) ; 6.22\left(1 \mathrm{H}, \mathrm{tt},{ }^{2} \mathrm{~J}_{\mathrm{HF}}=52.1 \mathrm{~Hz}\right) ; 6.90$ $\left(2 \mathrm{H}\right.$, app d); $7.19\left(2 \mathrm{H}\right.$, app d). ${ }^{19} \mathrm{~F}$ NMR $\left(400 \mathrm{MHz}, \mathrm{CDCl}_{3}\right):-117.1(2 \mathrm{~F}, \mathrm{~m}) ;-135.3\left(2 \mathrm{~F}, \mathrm{dt},{ }^{2} \mathrm{~J}_{\mathrm{HF}}=52.1 \mathrm{~Hz}\right)$. Anal. Calcd for $\mathrm{C}_{9} \mathrm{H}_{8} \mathrm{~F}_{4} \mathrm{O}_{4} \mathrm{~S}, \%$ : C, 37.51; H, 2.8; F, 26.37; S, 11.13. Found, \%: C, 37.54; H, 2.71; F, 26.01; S, 11.06.

4-Nitrophenyl-1,1,2,2-tetrafluoroethanesulfonate (6) was prepared following the procedure described for compound 5. Yield $3.35 \mathrm{~g}(92 \%)$. ${ }^{1} \mathrm{H}$ NMR $\left(400 \mathrm{MHz}, \mathrm{CDCl}_{3}\right): 6.27\left(1 \mathrm{H}, \mathrm{tt},{ }^{2} \mathrm{~J}_{\mathrm{HF}}=52.0 \mathrm{~Hz}\right) ; 7.49(2 \mathrm{H}$, app d); $8.35\left(2 \mathrm{H}\right.$, app d). ${ }^{19} \mathrm{~F}$ NMR $\left(400 \mathrm{MHz}, \mathrm{CDCl}_{3}\right)$ : $-115.9(2 \mathrm{~F}, \mathrm{~m}) ;-135.0\left(2 \mathrm{~F}, \mathrm{dt},{ }^{2} \mathrm{~J}_{\mathrm{HF}}=52.0 \mathrm{~Hz}\right)$. Anal. Calcd for $\mathrm{C}_{8} \mathrm{H}_{5} \mathrm{~F}_{4} \mathrm{NO}_{5} \mathrm{~S}, \%$ : C, 31.69; H, 1.66; F, 25.06. Found, \%: C, 31.73; H, 1.66; F, 25.10.

2,6-Dimethylphenyl-1,1,2,2-tetrafluoroethanesulfonate (7) was prepared following the procedure described for compound 5 using DBU instead of $\mathrm{Et}_{3} \mathrm{~N}$ and $\mathrm{HCF}_{2} \mathrm{CF}_{2} \mathrm{SO}_{2} \mathrm{~F}$ instead of $\mathrm{HCF}_{2} \mathrm{CF}_{2} \mathrm{SO}_{2} \mathrm{Cl}$. Yield $3.19 \mathrm{~g}(93 \%)$. ${ }^{1} \mathrm{H}$ NMR (400 MHz, $\left.\mathrm{CDCl}_{3}\right): 6.27\left(1 \mathrm{H}, \mathrm{tt},{ }^{2} \mathrm{~J}_{\mathrm{HF}}=52.0 \mathrm{~Hz}\right) ; 7.49\left(2 \mathrm{H}\right.$, app d); 8.35(2H, app d). ${ }^{19} \mathrm{~F} \mathrm{NMR}(400 \mathrm{MHz}$, $\left.\mathrm{CDCl}_{3}\right)$ : $-115.9(2 \mathrm{~F}, \mathrm{~m}) ;-135.0\left(2 \mathrm{~F}, \mathrm{dt},{ }^{2} \mathrm{~J}_{\mathrm{HF}}=52.0 \mathrm{~Hz}\right)$. Anal. Calcd for $\mathrm{C}_{10} \mathrm{H}_{10} \mathrm{~F}_{4} \mathrm{O}_{3} \mathrm{~S}, \%$ : C, 41.96; H, 3.52; F, 26.55; S, 11.2. Found, \%: C, 42.22; H, 3.74; F, 26.70; S, 11.15 .

Preparation of 4'-tert-Butyl-2-methylbiphenyl (Table 3, entry 1). In a drybox, a glass thick-walled pressure tube was charged with $0.15 \mathrm{~g}(1.1 \mathrm{mmol})$ of 2-methylphenylboronic acid, $0.314 \mathrm{~g}(1.0 \mathrm{mmol})$ of 4-tertbutylphenyl-1,1,2,2-tetrafluoroethane sulfonate, $0.5 \mathrm{~g}(3.3 \mathrm{mmol})$ of cesium fluoride, $0.6 \mathrm{~mL}$ of $0.1 \mathrm{M}$ solution of tricyclohexylphospine in THF, $1.5 \mathrm{~mL}$ of $0.033 \mathrm{M}$ solution of palladium acetate and $2 \mathrm{~mL}$ of THF. The tube was sealed, brought out of the drybox and heated at $55{ }^{\circ} \mathrm{C}$ for $16 \mathrm{~h}$. The reaction mixture was cooled to room temperature and volatiles were removed in vacuo. The residue was passed through a three-inch plug of silica gel with methylene chloride. Removal of the methylene chloride solution yielded a pale yellow liquid., which was further purified on a $30 \mathrm{~g}$ silica gel column using $2 \%$ ethylacetate/hexanes mixture to give $0.193 \mathrm{~g}(86 \%)$ of 4'tert-butyl-2-methylbiphenyl as a clear colorless liquid. ${ }^{1} \mathrm{H}$ NMR $\left(\delta, \mathrm{CDCl}_{3}\right): 1.37(\mathrm{~s}, 9 \mathrm{H}), 2.21(\mathrm{~s}, 3 \mathrm{H}), 7.21-7.28$ $(\mathrm{m}, 6 \mathrm{H}), 7.42(\operatorname{app~d}, 2 \mathrm{H}){ }^{1}$

4'-Nitro-2-methylbiphenyl (Table 3, entry 2). ${ }^{1} \mathrm{H}$ NMR $\left(\delta, \mathrm{CDCl}_{3}\right): 2.28(\mathrm{~s}, 3 \mathrm{H}), 7.22$ (app d, 1H), 7.27-7.36 $(\mathrm{m}, 3 \mathrm{H}), 7.50(\operatorname{app~d}, 2 \mathrm{H}), 8.30(\operatorname{app~d}, 2 \mathrm{H}){ }^{1}$

4'-Methoxy-2-methylbiphenyl (Table 3, entry 3). ${ }^{1} \mathrm{H} \mathrm{NMR}\left(\mathrm{\delta}, \mathrm{CDCl}_{3}\right): 2.33(\mathrm{~s}, 3 \mathrm{H}), 3.89(\mathrm{~s}, 3 \mathrm{H}), 7.00(\operatorname{app~d}$, $2 \mathrm{H}), 7.26-7.30(\mathrm{~m}, 6 \mathrm{H}){ }^{2}$

Preparation of $\mathrm{N}$-(4-tert-Butylphenyl)aniline (Table 4, entry 1). In a drybox, a glass thick-walled pressure tube was charged with $0.055 \mathrm{~mL}(0.6 \mathrm{mmoL})$ of aniline, $0.157 \mathrm{~g}(0.5 \mathrm{mmol})$ of 4-tert-butylphenyl-1,1,2,2tetrafluoroethane sulfonate, $0.067 \mathrm{~g}(0.7 \mathrm{mmol})$ of sodium tert-butoxide, $0.02 \mathrm{~g}(0.05 \mathrm{mmol})$ of 2(dicyclohexylphosphino)-2'-(N,N-dimethylamino)biphenyl, $0.023 \mathrm{~g} \quad(0.025 \mathrm{mmol})$ of palladium dibenzylideneacetone and $2 \mathrm{~mL}$ of toluene. The tube was sealed, brought out of the drybox and heated at $80{ }^{\circ} \mathrm{C}$ for $16 \mathrm{~h}$. The reaction mixture was cooled to room temperature, ether was added and the mixture was passed through a small plug of celite. The solvents were remove in vacuo. The crude product was purified on a $20 \mathrm{~g}$ silica gel column using $5 \%$ ethylacetate/hexanes. Removal of volatiles yielded $0.096 \mathrm{~g}(86 \%)$ of N-(4-tertbutylphenyl)aniline as a light brown oil. ${ }^{1} \mathrm{H} \mathrm{NMR}\left(\delta, \mathrm{CDCl}_{3}\right): 1.35(\mathrm{~s}, 9 \mathrm{H}), 5.65(\mathrm{br} \mathrm{s}, 1 \mathrm{H}), 6.92(\operatorname{app~t}, 1 \mathrm{H}, \mathrm{J}=$ $7.2 \mathrm{~Hz}), 7.07(\mathrm{~m}, 4 \mathrm{H}), 7.28(\operatorname{app~t}, 2 \mathrm{H}, \mathrm{J}=7.2 \mathrm{~Hz}), 7.33(\operatorname{app~d}, 2 \mathrm{H}, \mathrm{J}=8.7 \mathrm{~Hz}){ }^{3}$

$N$-(4-Nitrophenyl)aniline (Table 4, entry 2). ${ }^{1} \mathrm{H}$ NMR $\left(\delta, \mathrm{CDCl}_{3}\right): 6.26$ (br s, $\left.1 \mathrm{H}\right), 6.95$ (app d, $1 \mathrm{H}, \mathrm{J}=9.2$ $\mathrm{Hz}), 7.13-7.25(\mathrm{~m}, 3 \mathrm{H}), 7.40(\operatorname{app~t}, 2 \mathrm{H}, \mathrm{J}=7.9 \mathrm{~Hz}), 8.13(\operatorname{app~d}, 2 \mathrm{H}, \mathrm{J}=9.2 \mathrm{~Hz}){ }^{4}$

N-(4-Methoxyphenyl)aniline (Table 4, entry 3). ${ }^{1} \mathrm{H}$ NMR $\left(\delta, \mathrm{CDCl}_{3}\right): 3.81(\mathrm{~s}, 3 \mathrm{H}), 5.48$ (br s, 1H), 6.81-6.94 $(\mathrm{m}, 5 \mathrm{H}), 7.09(\operatorname{app~d}, 2 \mathrm{H} . \mathrm{J}=8.8 \mathrm{~Hz}), 7.22(\operatorname{app~t}, 2 \mathrm{H}, \mathrm{J}=7.7 \mathrm{~Hz}){ }^{5}$

\footnotetext{
${ }^{1}$ Wolfe, J. P.; Singer, R. A.; Yang, B. H.; Buchwald, S. L., J. Am. Chem. Soc. 1999, 121(41), 9550-9561.

${ }^{2}$ Lipshutz, B. H.; Siegmann, K.; Garcia, E.; Kayser, F., J. Am. Chem. Soc. 1993, $115(20)$, 9276-9282.

${ }^{3}$ Wolfe, J. P.; Buchwald, S. L., J. Org. Chem. 2000, 65(4), 1144-1157.

${ }^{4}$ Kataoka, N.; Shelby, Q.; Stambuli, J. P.; Hartwig, J. F., J. Org. Chem. 2002, 67(16), 5553-5566.

${ }^{5}$ Louie, J.; Driver, M. S.; Hamann, B. C.; Hartwig, J. F., J. Org. Chem. 1997, 62(5), 1268-1273.
} 
Reaction of 4-methoxyphenyl tetraflate with styrene (Table 5, entry 3). In a drybox, a thick-walledd glass pressure tube was charged with $0.909 \mathrm{~mL}$ of styrene, $0.144 \mathrm{~g}(0.5 \mathrm{mmol})$ of 4-methoxyphenyl-1,1,2,2tetrafluoroethane sulfonate, $0.084 \mathrm{~mL}(0.6 \mathrm{mmol})$ of triethylamine, $0.02 \mathrm{~g}(0.05 \mathrm{mmol})$ of $1,3-$ bis(diphenylphosphino)propane, $0.023 \mathrm{~g}(0.025 \mathrm{mmol})$ of palladium dibenzylideneacetone and $1.5 \mathrm{~mL}$ of dimethylformamide. The tube was sealed, brought out of the drybox and heated at $80^{\circ} \mathrm{C}$ for $16 \mathrm{~h}$. The reaction was cooled to room temperature and ether was added. The ether solution was washed two times with $3 \mathrm{M} \mathrm{HCl}$, three times with water and two times with brine. The organic layer was dried over $\mathrm{MgSO}_{4}$ and concentrated. The crude product was purified on a silica gel column using $100 \%$ hexanes, $2 \%$ ethylacetate/hexanes and $10 \%$ ethylacetate/hexanes to yield $67.1 \mathrm{mg}(63.8 \%)$ white solid product. ${ }^{1} \mathrm{H}$ NMR shows the product to be a 50:50 mixture of (E)- $\beta$-phenyl-4-methoxystyrene and $\alpha$-phenyl-4-methoxystyrene. ${ }^{6}{ }^{1} \mathrm{H}$ NMR $\left(\delta, \mathrm{CD}_{3} \mathrm{CN}\right): 3.82(\mathrm{~s}, 3 \mathrm{H}$, $\left.\mathrm{OCH}_{3}\right), 3.83\left(\mathrm{~s}, 3 \mathrm{H}, \mathrm{OCH}_{3}\right), 5.35(\mathrm{~d}, 1 \mathrm{H}, \mathrm{J}=1.3 \mathrm{~Hz}), 5.39(\mathrm{~d}, 1 \mathrm{H}, \mathrm{J}=1.3 \mathrm{~Hz}), 6.86(\operatorname{app~d}, 2 \mathrm{H}, \mathrm{J}=8.7 \mathrm{~Hz}), 6.90$ $(\operatorname{app~d}, 2 \mathrm{H}, \mathrm{J}=8.5 \mathrm{~Hz}), 6.97(\mathrm{~d}, 1 \mathrm{H}, \mathrm{J}=16.1 \mathrm{~Hz},-\mathrm{CH}=\mathrm{CH}-), 7.06(\mathrm{~d}, 1 \mathrm{H}, \mathrm{J}=16.1 \mathrm{~Hz}), 7.2-7.35(\mathrm{~m}, 10 \mathrm{H}), 7.45$ (app d, 2H, J = 8.9 Hz), $7.49(\operatorname{app~d,~2H,~J~=~7.8~Hz).~}$

Reaction of 4-tert-butylphenyl tetraflate with styrene (Table 5, entry 1). A 6:1 mixture of $\beta$-(E)-isomer to $\alpha$ isomer was isolated. The two isomers were identified by the characteristic signals of $(E) \mathrm{CH}=\mathrm{CH}$ and $\mathrm{C}=\mathrm{CH}_{2}$ groups in ir ${ }^{1} \mathrm{H}$ NMR, respectively. ${ }^{7}{ }^{1} \mathrm{H}$ NMR $\left(\delta, \mathrm{CDCl}_{3}\right): 1.33(\mathrm{~s}, 9 \mathrm{H}), 5.40(\mathrm{~d}, \mathrm{~J}=1.3 \mathrm{~Hz}), 5.45(\mathrm{~d}, \mathrm{~J}=1.3 \mathrm{~Hz})$, 7.05 (AB system, 2H, J = 16.3 Hz), 7.2-7.5 (m, 9H).

Reaction of 4-nitrophenyl tetraflate with styrene (Table 5, entry 2). Only (E)- $\beta$-phenyl-4-nitrostyrene was isolated. ${ }^{1} \mathrm{H}$ NMR $\left(\delta, \mathrm{CDCl}_{3}\right): 7.2-7.35(\mathrm{~m}, 3 \mathrm{H}), 7.30(\mathrm{~d}, 1 \mathrm{H}, \mathrm{J}=16.8 \mathrm{~Hz}), 7.42(\mathrm{~d}, 1 \mathrm{H}, \mathrm{J}=16.8 \mathrm{~Hz}), 7.57(\mathrm{app}$ d, $2 \mathrm{H}, \mathrm{J}=8 \mathrm{~Hz}), 7.77(\operatorname{app} \mathrm{d}, 2 \mathrm{H}, \mathrm{J}=8.7 \mathrm{~Hz}), 8.14(\operatorname{app} \mathrm{d}, 2 \mathrm{H}, \mathrm{J}=8.7 \mathrm{~Hz}){ }^{8}$

${ }^{6}$ Fristrup, P.; Le Quement, S.; Tanner, D.; Norrby, P.-O., Organometallics 2004, 23(26), 6160-6165.

7 1,2-Isomer: Smith, J. G.; Oliver, E.; Boettger, T. J., Organometallics 1983, 2(11), 1577-82.; 1,1-isomer: Hansen, A. L.; Ebran, J.-P.; Gogsig, T. M.; Skrydstrup, T., Chem. Comm. 2006, 4137-4139..

${ }^{8}$ Zhang, Z.; Wang, Z., J. Org. Chem. 2006, $71(19), 7485-7487$. 
${ }^{1} \mathrm{H}$ and ${ }^{19} \mathrm{~F}$ NMR of $\mathrm{HCF}_{2} \mathrm{CF}_{2} \mathrm{SO}_{2} \mathrm{Cl}$ (1)

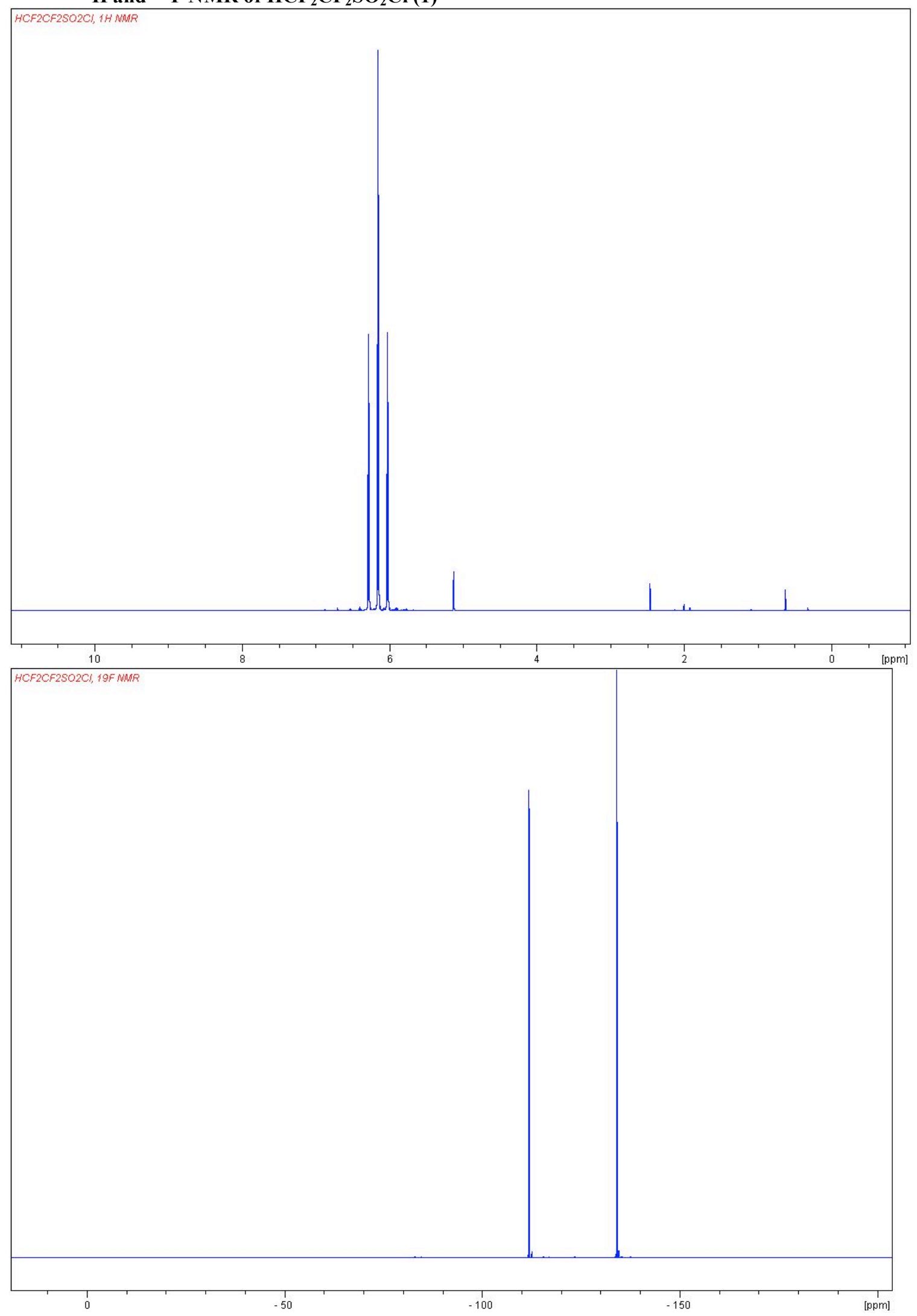


${ }^{1} \mathrm{H}$ and ${ }^{19} \mathrm{~F}$ NMR of $\mathrm{HCF}_{2} \mathrm{CF}_{2} \mathrm{SO}_{2} \mathrm{~F}$ (2)

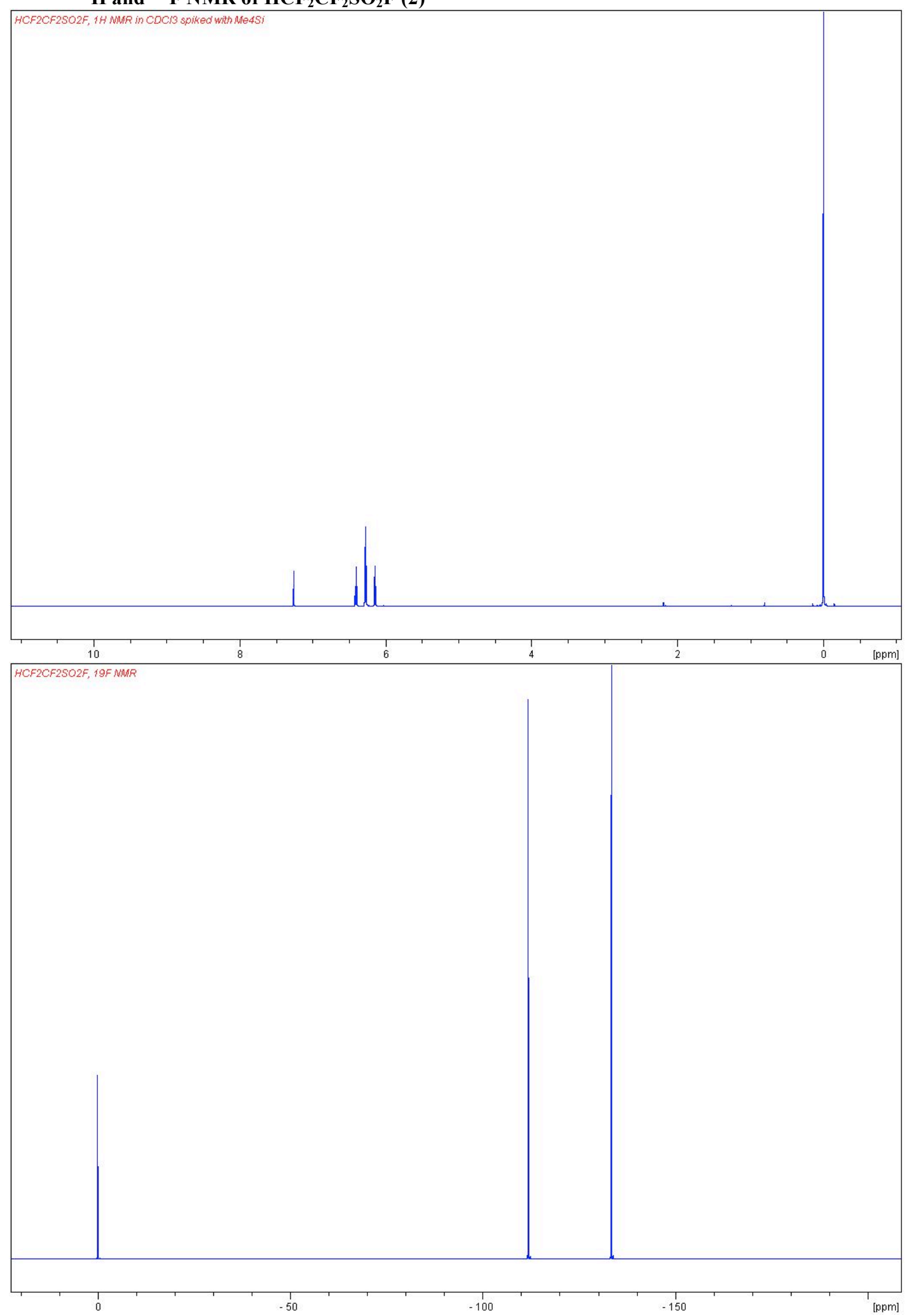


${ }^{1} \mathrm{H}$ and ${ }^{19} \mathrm{~F}$ NMR of $\left(\mathrm{HCF}_{2} \mathrm{CF}_{2} \mathrm{SO}_{2}\right)_{2} \mathrm{O}(3)$, small impurity is $\mathrm{HCF}_{2} \mathrm{CF}_{2} \mathrm{SO}_{2} \mathrm{OCF}_{2} \mathrm{CF}_{2} \mathrm{H}$

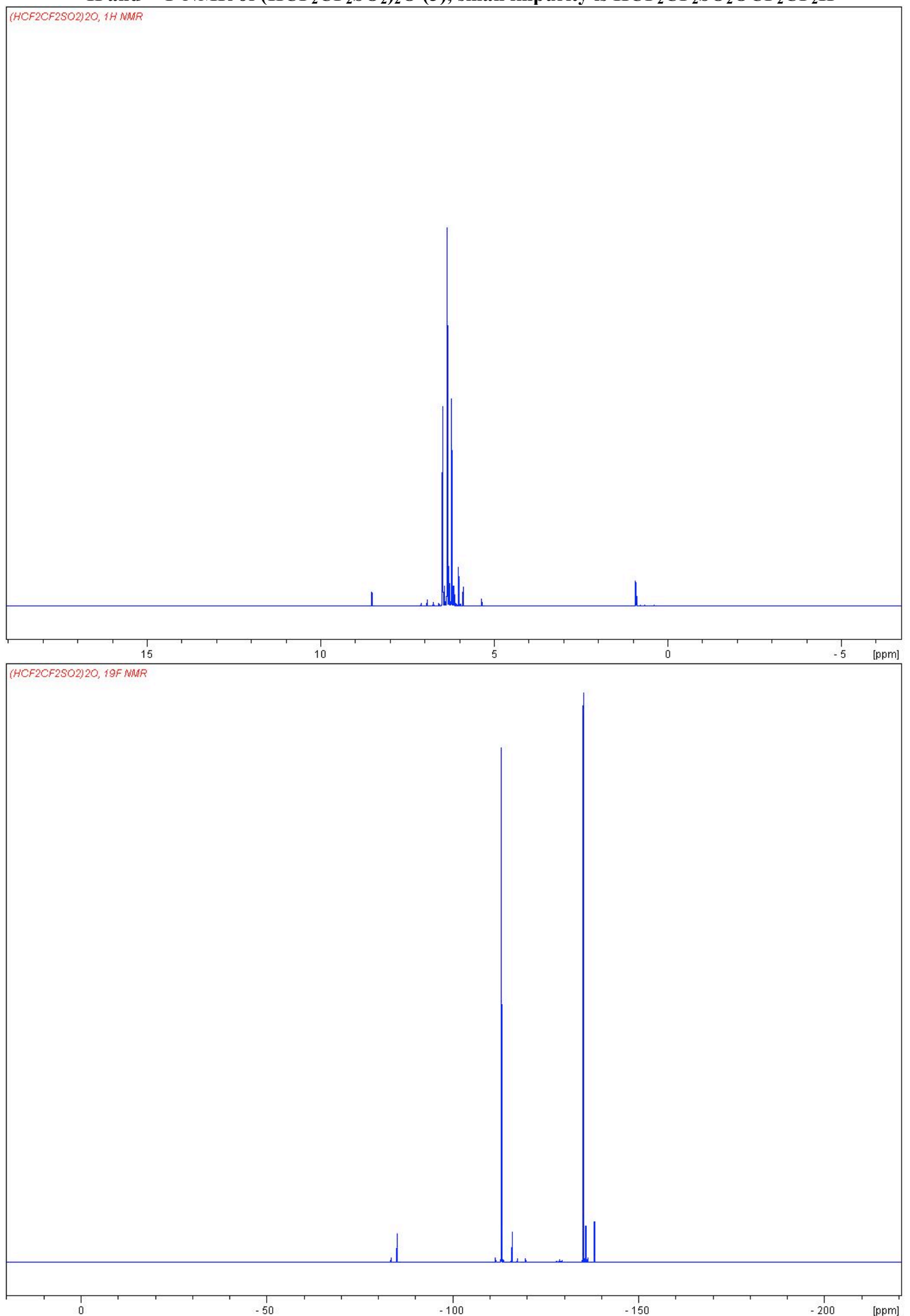


${ }^{1} \mathrm{H}$ and ${ }^{19} \mathrm{~F}$ NMR of 4-MeO- $\mathrm{C}_{6} \mathrm{H}_{4} \mathrm{O}-\mathrm{SO}_{2} \mathrm{CF}_{2} \mathrm{CF}_{2} \mathrm{H}$ (4)

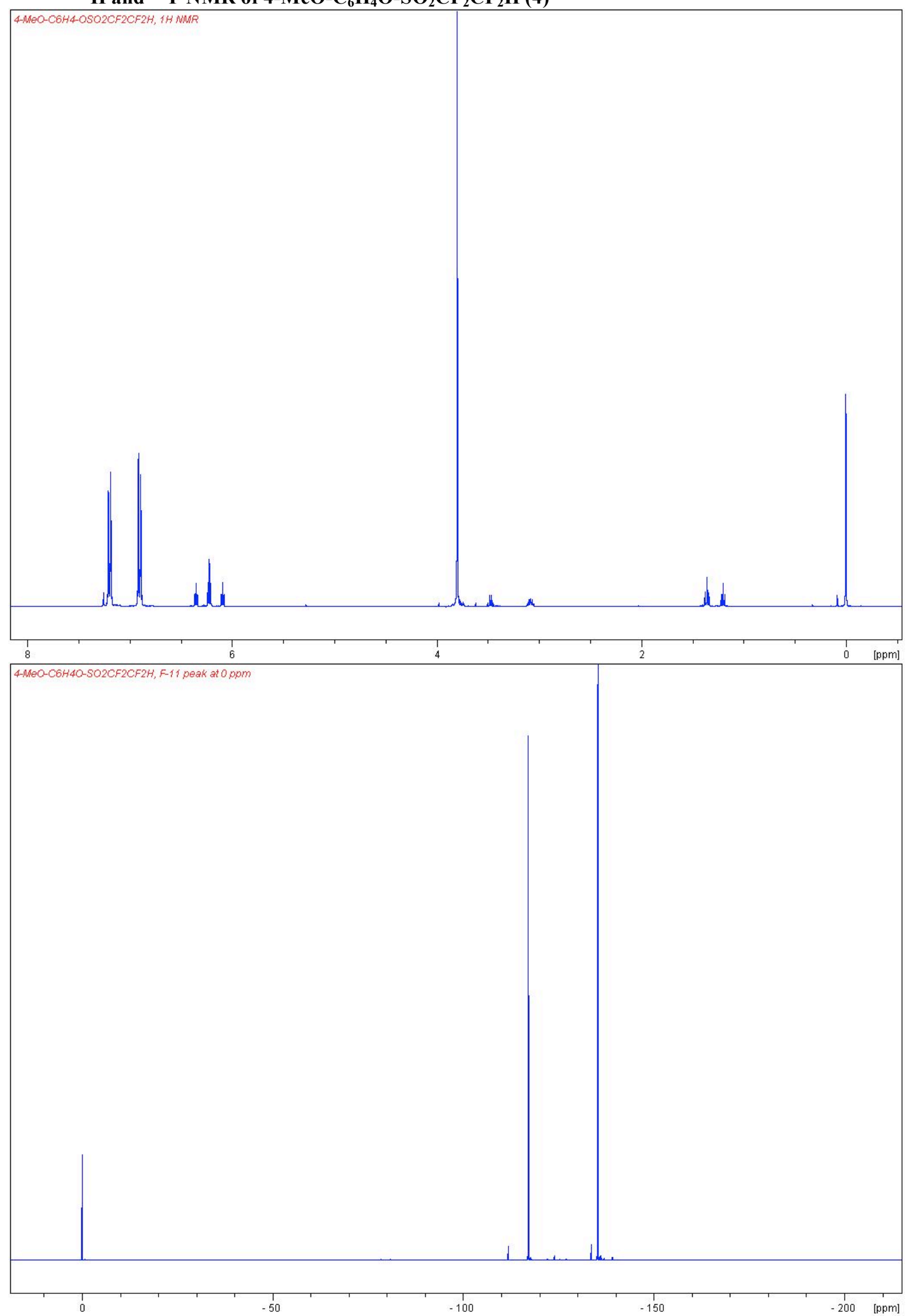


${ }^{1} \mathrm{H},{ }^{13} \mathrm{C}$ and ${ }^{19} \mathrm{~F}$ NMR of 4-t-Bu- $\mathrm{C}_{6} \mathrm{H}_{4} \mathrm{O}-\mathrm{SO}_{2} \mathrm{CF}_{2} \mathrm{CF}_{2} \mathrm{H}$ (5).

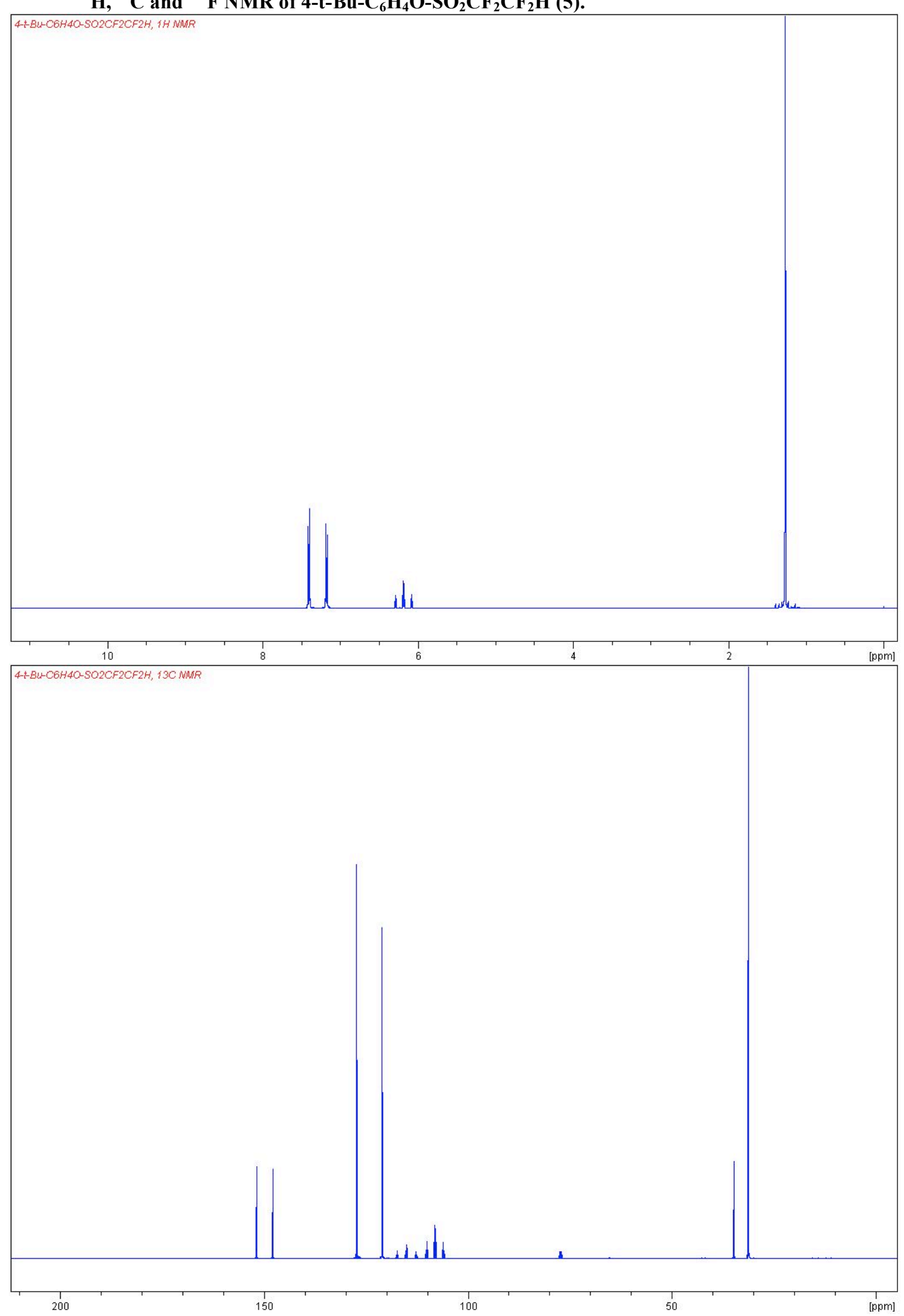




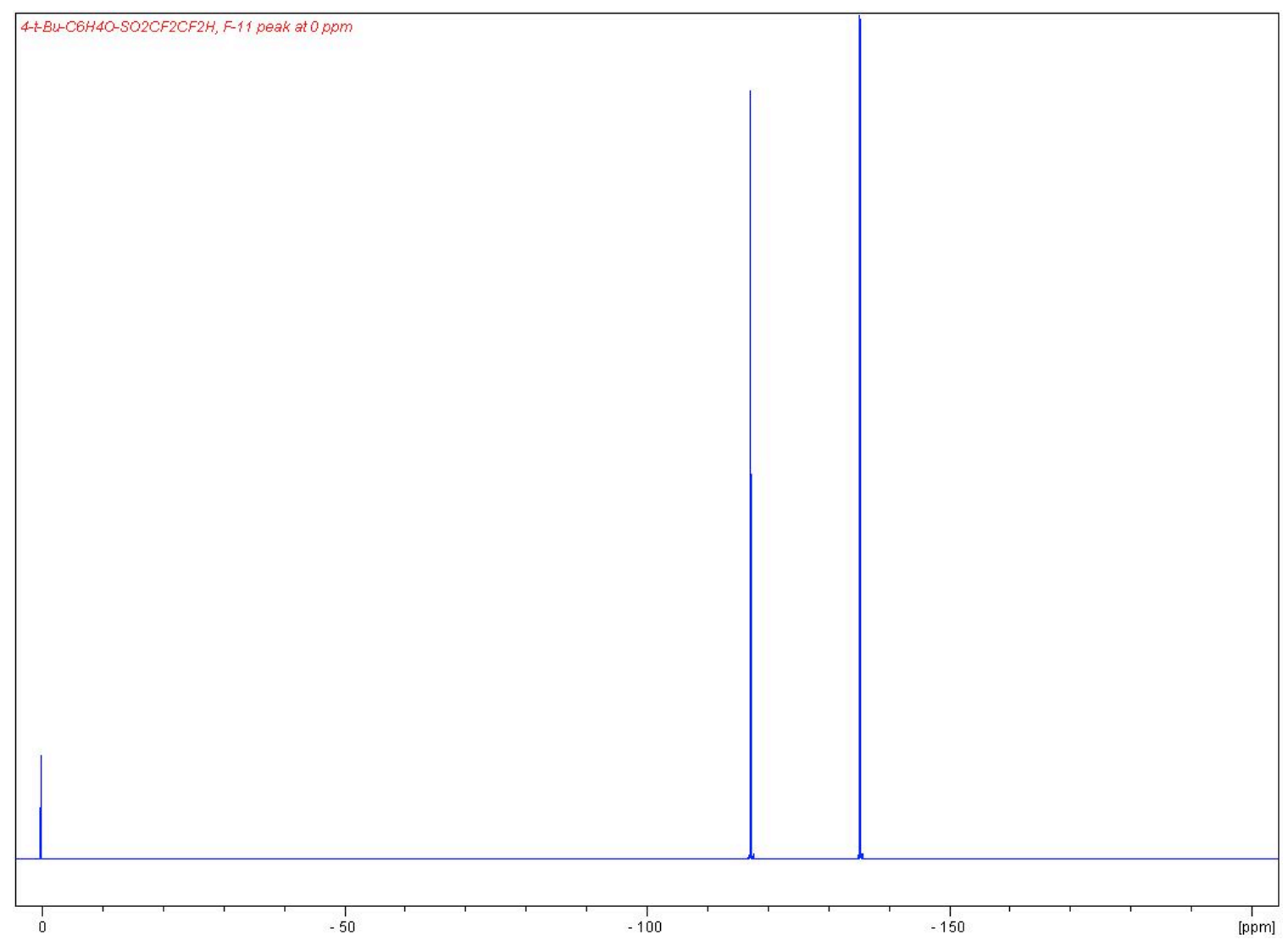


${ }^{1} \mathrm{H}$ and ${ }^{19} \mathrm{~F}$ NMR of $4-\mathrm{NO}_{2}-\mathrm{C}_{6} \mathrm{H}_{4} \mathrm{O}-\mathrm{SO}_{2} \mathrm{CF}_{2} \mathrm{CF}_{2} \mathrm{H}(6)$.

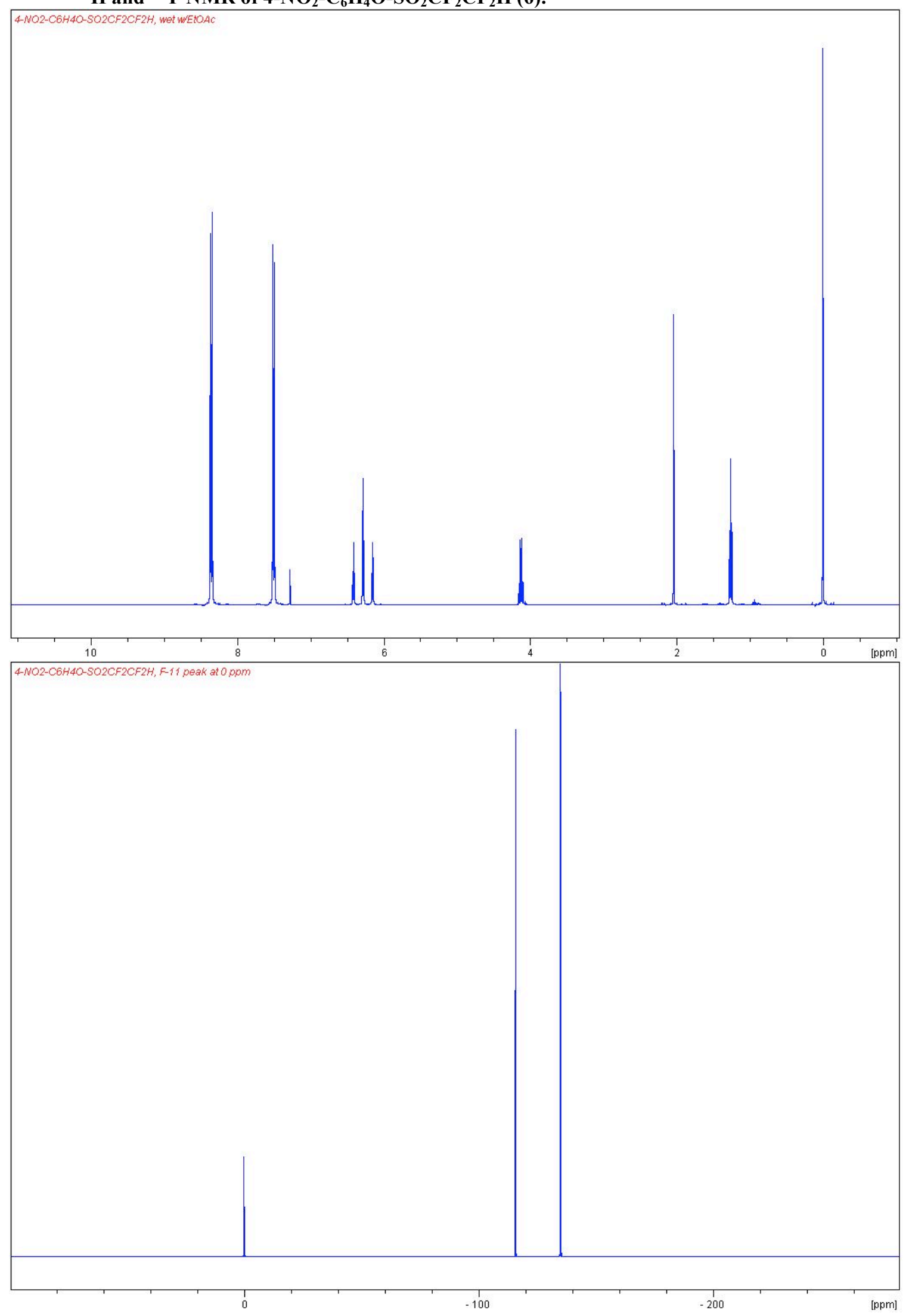


${ }^{1} \mathrm{H}$ and ${ }^{19} \mathrm{~F}$ NMR of 2,6- $-\mathrm{Me}_{2}-\mathrm{C}_{6} \mathrm{H}_{3} \mathrm{O}-\mathrm{SO}_{2} \mathrm{CF}_{2} \mathrm{CF}_{2} \mathrm{H}$ (7).

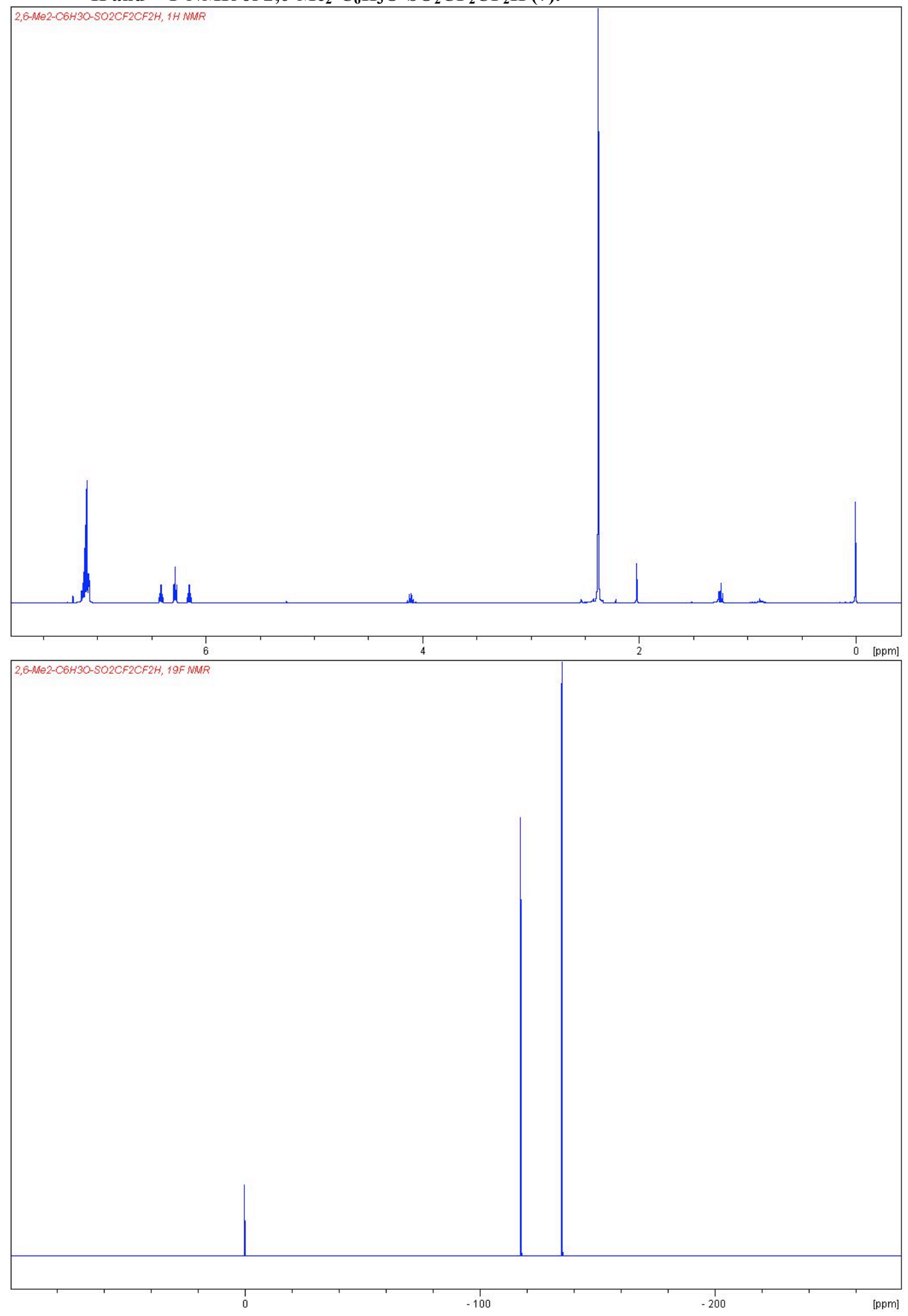

